\title{
Comparison of C-reactive protein and C-reactive protein-to-albumin ratio in predicting mortality among geriatric coronavirus disease 2019 patients
}

\author{
Rohat $\mathrm{Ak}^{1 *}$ (D), Fatih Doğanay² (D), Erdal Yilmaz ${ }^{1}$
}

\begin{abstract}
SUMMARY
OBJECTIVE: The aim of this study was to evaluate and compare C-reactive protein and C-reactive protein-to-albumin ratio performances in predicting mortality of geriatric patients who visited the emergency department.

METHODS: The data of patients with COVID-19 and aged 65 years and above, who visited emergency department during the study period, were retrospectively analyzed. The data were obtained from an electronic-based hospital information system. The area under the receiver operating characteristic curve and the area under the curve were used to assess each cutoff value discriminatory for predicting mortality.

RESULTS: The mean age of the population included in this study was 76 (71-82) years, while $52.7 \%$ were males. The sensitivity, specificity, and area under the curve values for C-reactive protein in terms of mortality were calculated as 71.01, 52.34, and 0.635\%, respectively, while the sensitivity, specificity, and area under the curve values for C-reactive protein-to-albumin ratio were calculated as 75.74, 47.66, and 0.645\%, respectively $(p<0.001)$. In the pairwise comparison for the receiver operating characteristic curves of C-reactive protein and C-reactive protein-to-albumin ratio, no statistically significant difference was found.

CONCLUSIONS: Geriatric patients are the "most vulnerable" patient group against the COVID-19. In this study, both C-reactive protein and C-reactive protein-to-albumin ratio were found to be successful in predicting mortality for geriatric COVID-19 patients.

KEYWORDS: Albumins. COVID-19. C-reactive protein. Geriatrics. Mortality.
\end{abstract}

\section{INTRODUCTION}

It has been more than a year and a half since the first case was emerged in Wuhan (China), in December 2019'. The novel coronavirus, the severe acute respiratory syndrome coronavirus 2 (SARS-CoV-2), caused an epidemic described as coronavirus disease 2019 (COVID-19)2. The spectrum of COVID-19 ranges from possibly asymptomatic patients to acute respiratory distress syndrome, which leads to severe progressive pneumonia and death ${ }^{3,4}$.

There is obvious evidence that geriatric patients have a higher risk of mortality from COVID-195. Elderly individuals are more susceptible to the outcomes of SARS-CoV-2 infection than younger people due to weaker immune systems, comorbidities, and the presence of underlying conditions $s^{6,7}$. Therefore, early identification of patients who will require critical care is vital for the geriatric patient population.

It has been understood that the C-reactive protein (CRP) level is associated with inflammation, and its concentration in the blood is not influenced by age, gender, or physical condition ${ }^{8}$. The CRP is a well-known index of serious pulmonary infections, and it has been reported to be positively correlated with severity in COVID-19 disease 9 . Albumin is an important component of serum proteins and is an indicator of systemic inflammation ${ }^{10}$. Low albumin is shown to have the poor nutritional status and liver and kidney dysfunction. It is further accepted as an independent indicator of poor survival in critically ill patients ${ }^{11}$. Low albumin levels in COVID-19 patients were found to be associated with a poor prognosis ${ }^{12}$. In a recent study, the ratio of these two inflammatory markers to each other was reported to be associated with mortality in CRP-to-albumin ratio (CAR) and COVID-19 patients ${ }^{13}$.

The aim of this study was to examine the relationship between CAR and mortality at the time of admission in patients with COVID-19 who visited the emergency department (ED).

\section{METHODS}

This single-center, retrospective, and observational study was carried out in the ED of a tertiary care teaching hospital between

\footnotetext{
${ }^{1}$ Kartal Dr. Lütfi Kırdar City Hospital, Department of Emergency Medicine - Istanbul, Turkey.

${ }^{2}$ Edremit Public Hospital, Department of Emergency Medicine - Balıkesir, Turkey.

*Corresponding author: rohatakmd@gmail.com

Conflicts of interest: the authors declare there is no conflicts of interest. Funding: none.

Received on August 20, 2021. Accepted on September 24, 2021.
} 
February 1, 2021, and April 1, 2021. The institutional review board approved the analysis and issued a waiver of consent (Ethics Committee ruling number: 2021/514/200/28, dated: April 28, 2021). During the two-month period assigned for this study, CRP and albumin tests were requested at the time of admission to the $\mathrm{ED}$, and the patients who were aged 65 years and above and hospitalized were included in this study. The diagnosis of COVID-19 was determined according to the World Health Organization (WHO) guidelines. This study included only patients who had positive results in the reverse transcriptase polymerase chain reaction (RT-PCR) test of nasal and pharyngeal swab samples ${ }^{14}$. Patients with negative RT-PCR test results, patients with deficient CRP and/or albumin values, patients transferred from another hospital, patients who died or were discharged from the ED, and patients aged below 65 years were excluded from this study.

The following data were collected from each patient scanning the hospital-based electronic data recording system: age, gender, PCR test results, comorbidities [chronic obstructive pulmonary disease (COPD), coronary artery disease (CAD), hypertension (HT), diabetes mellitus (DM), congestive heart failure (CHF), atrial fibrillation (AF), chronic neurological disease (CND), and chronic renal failure (CRF)], and laboratory results [complete blood count (CBC), CRP, and albumin levels]. If an eligible patient was admitted more than once during the study period, only the most initial visit was included in the analysis. The most abnormal values were registered in patients who had more than one laboratory test in the ED. CAR $(\mathrm{mg} / \mathrm{g})$ values were calculated by dividing the CRP $(\mathrm{mg} / \mathrm{L})$ value by the albumin $(\mathrm{g} / \mathrm{L})$ value. The study data were registered into an Excel database (Microsoft Inc., Richmond, WA, USA) and analyzed by the first researcher. After data analysis, other researchers performed quality improvement feedback. The primary study outcome was the patient's mortality, and the survival follow-up was assessed 28 days after admission.

\section{Statistical analysis}

Statistical analysis was performed using IBM SPSS Statistics version 26.0 and MedCalc Statistical Software version 19.0.6. The Mann-Whitney U test was used for continuous data analysis, and the chi-square test was used for categorical data analysis. The continuous data were reported as median and interquartile range (IQR). The categorical data were presented as frequency and percentage (Tables 1 and 2). A $\mathrm{p}<0.05$ was considered statistically significant.

Receiver operating characteristic (ROC) analysis was performed using the DeLong method to evaluate the prognostic performance of CRP and $\mathrm{CAR}^{15}$. The area under the curve
(AUC) was calculated to evaluate the prognostic performance of the CRP and CAR parameters. The Youden's J index (YJI) analysis was used to calculate the sensitivity, specificity, positive predictive value (PPV), negative predictive value (NPV), and the threshold value at the highest $\mathrm{AUC}^{16}$.

\section{RESULTS}

This study was conducted using data from a total of 404 patients, of which 191 were women and 213 were men. There were 235 patients in the survivor group and 169 patients in the nonsurvivor group. The median age of the population included in this study was 76 (71-82) years, with a minimum age of 65 and a maximum age of 102 . The median age was 75 (70-81) years for the survivor group and $78(72-84)$ years for the nonsurvivor group.

When the impacts of the chronic diseases on the COVID19 prognosis were examined, a significant difference was found between the groups for DM, CHF, and CRF, while no significant difference was found between the groups for COPD, HT, $\mathrm{CAD}, \mathrm{AF}$, and CND (Table 1). When the impacts of laboratory parameters on the COVID-19 prognosis were analyzed, a significant difference was found between the groups for white blood cells (WBCs), neutrophils, lymphocytes, CRP, albumin, and CAR, while no significant difference was found between the groups for hemoglobin and platelets.

The predictive values of the CRP and the CAR in terms of in-hospital mortality were analyzed by ROC analysis. The sensitivity, specificity, PPV, NPV, AUC, and YJI values of the CRP were calculated as $71.01,52.34,51.7,71.5,0.635$, and $0.23 \%$, respectively $(\mathrm{p}<0.001)$, in terms of in-hospital mortality (Figure 1 and Table 2). The sensitivity, specificity, PPV, NPV, AUC, and YJI values of the CAR were calculated as 75.74 , $47.66,51.0,73.2,0.645$, and $0.23 \%$, respectively, in terms of in-hospital mortality.

When the ROC curves of the CRP and the CAR were compared, the difference between AUCs was calculated as 0.0104, and the p-value was 0.056 . No statistically significant difference was found in the pairwise comparison of the ROC curves.

\section{DISCUSSION}

In this study, geriatric COVID-19 patients who were admitted to the ED were examined in two groups as survivor and nonsurvivor. It was concluded that the mortality group had significantly higher CAR and CRP values at the time of admission. However, no significant difference was found between CRP and CAR. 
Depending on the increase in human life span, changes occur in many physiological systems of geriatric individuals. The immune system may be suppressed due to aging. This can further be defined by the reduction in T cells and T-cell receptors produced in the thymus. These changes increase the risk of infections and cause an increase in mortality rates of elderly individuals ${ }^{17}$. This weak situation that appears with aging has additionally shown its impact on the COVID-19 epidemic period. For instance, Italy had an overall mortality rate of $12.6 \%$ due to the epidemic. Furthermore, the mortality rate was found to increase significantly with age: it increased to $<1 \%$ in patients below 50 years of age, $2.6 \%$ in the fifth decade, $9.8 \%$ in the sixth decade, $24.2 \%$ in the seventh decade, and $29.0 \%$ in the eighth decade 5 .

The COVID-19 epidemic had a significant impact on geriatric individuals, and advanced age was reported to be an independent risk factor for the disease ${ }^{18}$. Therefore, early risk prediction tools in ED admissions of geriatric patients have been an important research topic in the literature. The inflammatory reaction plays a significant role in the pathophysiology of

Table 1. Demographic and comorbidity data of the study population.

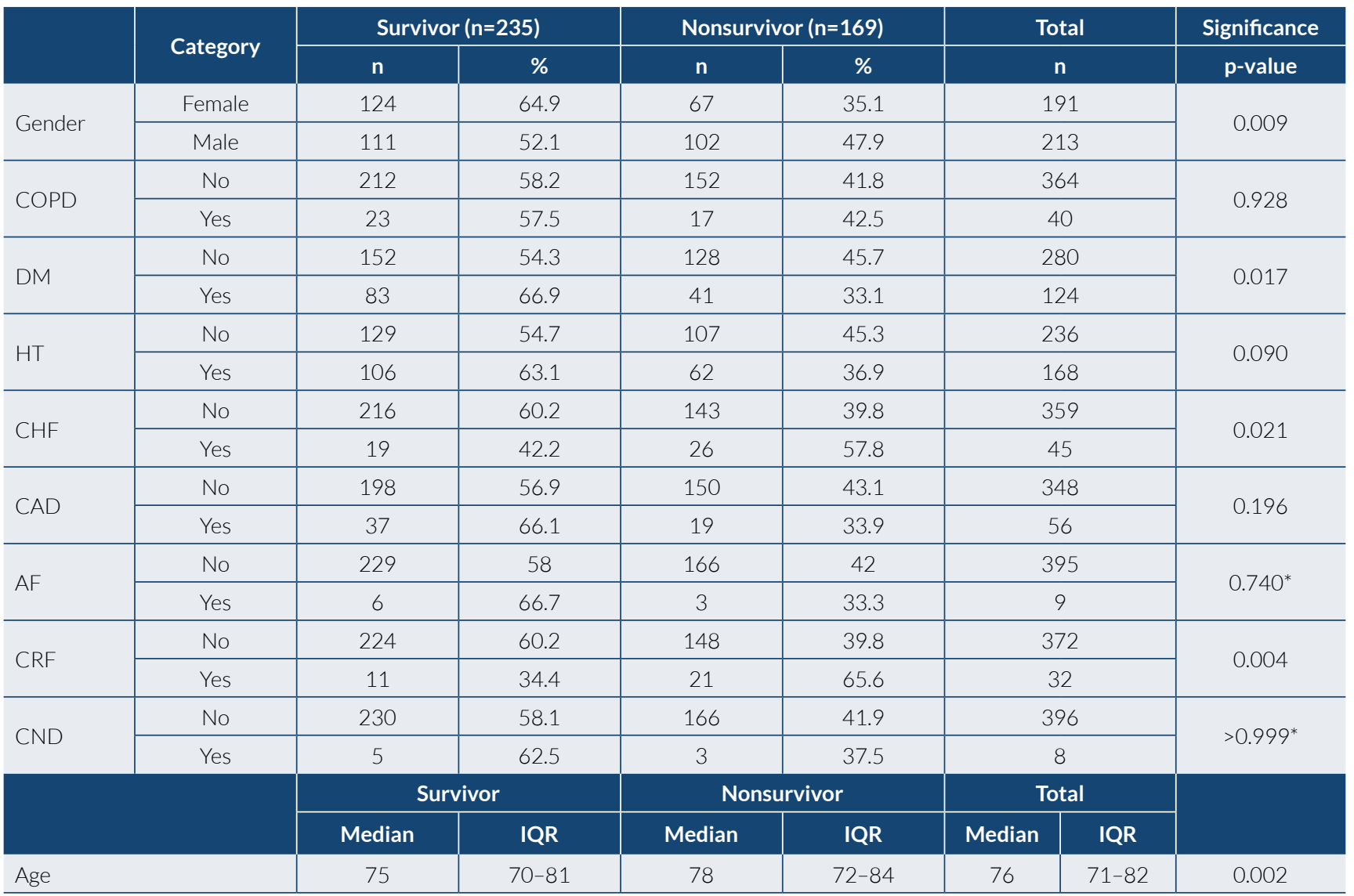

COPD: chronic obstructive pulmonary disease; DM: diabetes mellitus; HT: hypertension; CHF: congestive heart failure; CAD: coronary artery disease; AF: atrial fibrillation; CRF: chronic renal failure; CND: chronic neurological disease. ${ }^{*}$ Fisher's exact test.

Table 2. Predictive performance of C-reactive protein and C-reactive protein-to-albumin ratio in terms of severity in COVID-19 patients.

\begin{tabular}{|c|c|c|c|c|c|c|c|c|}
\hline & $\begin{array}{l}\text { Sensitivity } \\
(95 \% \mathrm{CI})\end{array}$ & $\begin{array}{l}\text { Specificity } \\
(95 \% \mathrm{CI})\end{array}$ & $\begin{array}{c}\text { PPV } \\
(95 \% \mathrm{CI})\end{array}$ & $\begin{array}{c}\text { NPV } \\
(95 \% \mathrm{Cl})\end{array}$ & $\begin{array}{c}\text { AUC } \\
(95 \% \mathrm{Cl})\end{array}$ & $\begin{array}{c}\text { YJI } \\
(95 \% \mathrm{CI})\end{array}$ & $\begin{array}{c}\text { Criterion of } \\
\text { YJI }\end{array}$ & p-value* \\
\hline CAR & $\begin{array}{c}75.74 \\
(68.6-82.0)\end{array}$ & $\begin{array}{c}47.66 \\
(41.1-54.3)\end{array}$ & $\begin{array}{c}51.0 \\
(47.3-54.7)\end{array}$ & $\begin{array}{c}73.2 \\
(67.0-78.6)\end{array}$ & $\begin{array}{c}0.645 \\
(0.596-0.692)\end{array}$ & $\begin{array}{c}0.23 \\
(0.14-0.31)\end{array}$ & $>1.54$ & $<0.001$ \\
\hline $\begin{array}{l}\text { CRP } \\
\text { (mg/L) }\end{array}$ & $\begin{array}{c}71.01 \\
(63.5-77.7)\end{array}$ & $\begin{array}{c}52.34 \\
(45.7-58.9)\end{array}$ & $\begin{array}{c}51.7 \\
(47.6-55.8)\end{array}$ & $\begin{array}{c}71.5 \\
(65.8-76.6)\end{array}$ & $\begin{array}{c}0.635 \\
(0.586-0.682)\end{array}$ & $\begin{array}{c}0.23 \\
(0.14-0.32)\end{array}$ & $>62.3$ & $<0.001$ \\
\hline
\end{tabular}

CI: confidence interval; PPV: positive predictive value; NPV: negative predictive value; AUC: area under the curve; YJI: Youden's J index; CAR: C-reactive proteinto-albumin ratio; CRP: C-reactive protein. ${ }^{*}$ In the pairwise comparison of the Receiver operating characteristic curves of C-reactive protein and C-reactive protein-to-albumin ratio, $p=0.056$. 


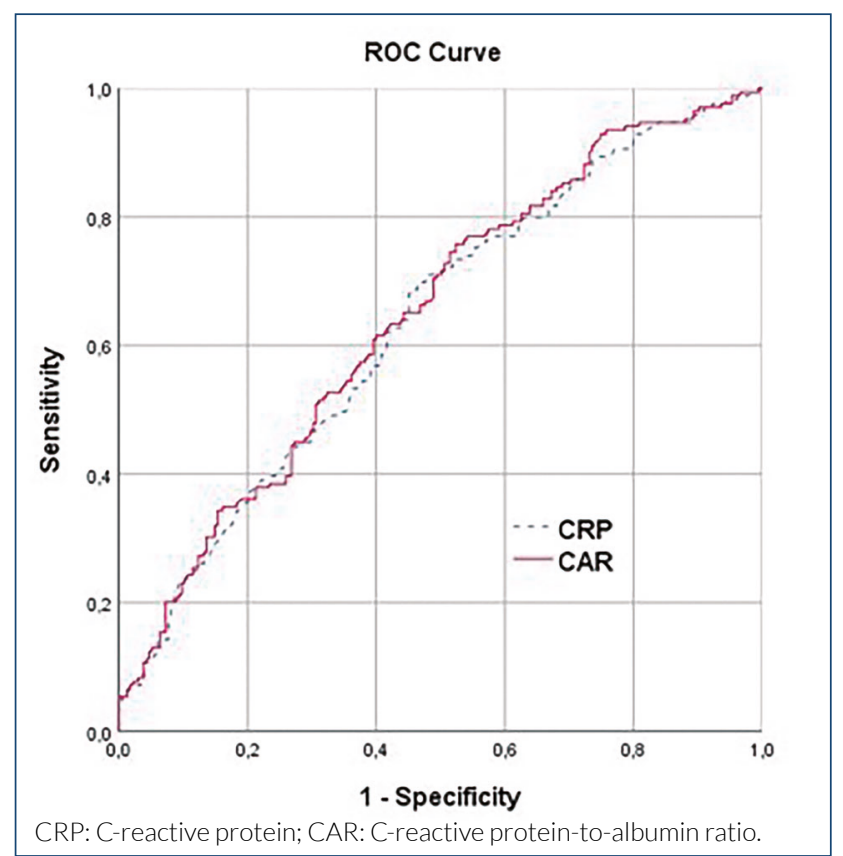

Figure 1. Receiver operating characteristic curves of C-reactive protein and $\mathrm{C}$-reactive protein-to-albumin ratio for mortality prediction in COVID-19 patients.

COVID-19 ${ }^{19}$. Therefore, inflammatory markers such as CRP have been studied as prognostic indicators of COVID- $19^{20}$. It has been shown that albumin, which is expected to decrease in inflammatory conditions, also decreases in severe COVID19 patients $^{21}$. El-Shabrawy et al. examined the importance of biomarkers in predicting the prognosis of COVID-19 in their study. In this study, a total of 116 patients were studied in two groups as severe and nonsevere, and it was concluded that a high CAR value could be used as an independent marker during the prediction of 30-day mortality in COVID-19 patients $^{13}$. In the light of this information, the results of our study were found to be in line with the literature.

In this study, the relationship between patients' comorbidities and their mortality was also examined. A significant

\section{REFERENCES}

1. Guan WJ, Ni ZY, Hu Y, Liang WH, Ou CQ, He JX, et al. Clinical Characteristics of Coronavirus Disease 2019 in China. N Engl J Med. 2020;382(18):1708-20.https://doi.org/10.1056/NEJMoa2002032

2. Seyhan AU, Doganay F, Yilmaz E, Topal NP, Ak R. Investigation of QT Prolongation with Hydroxychloroquine and Azithromycin for the Treatment ofCOVID-19.J Coll Physicians Surg Pak.2020;30(10):153-7. https://doi.org/10.29271/jcpsp.2020.supp2.S153

3. Chen N, Zhou M, Dong X, Qu J, Gong F, Han Y, etal. Epidemiological and clinical characteristics of 99 cases of 2019 novel coronavirus pneumonia in Wuhan, China: a descriptive study. Lancet. 2020;395(10223):50713. https://doi.org/10.1016/S0140-6736(20)30211-7 correlation was found among DM, CHF, CRF, and mortality. These outcomes are not unexpected. One of the most common comorbidities in geriatric individuals with COVID-19 infection is $\mathrm{DM}^{22}$. In a meta-analysis, the prevalence of diabetes in hospitalized patients was $9.7 \%$. In a large-scale worldwide observational study including 169 hospitals and approximately 9000 patients from three continents, CHF was found to be an independent predictor of in-hospital mortality ${ }^{23}$. The mortality rate associated with pneumonia in patients with CRF appears to be 14-16 times higher than that in the general population $^{24}$. In a meta-analysis including 1389 COVID-19 patients, a significant association was found between the CRF and severe COVID-1925.

As with any retrospective study, this study has some limitations. First, our sample size was small, thus limiting the potential of our analysis. In addition, we conducted the study at a single institution; therefore, the findings may not be representative of the general population of COVID-19 patients aged $\geq 65$ years. Conclusively, the study focused on patient mortality. Accordingly, we cannot predict other related outcomes for the geriatric population, such as patients' persistent oxygen demand or requirement for transfer to a care center.

\section{CONCLUSIONS}

Geriatric patients are the patient group with the highest risk of poor outcome for COVID-19. This study demonstrated that the CRP and CAR geriatric patient population had good predictive performance in predicting mortality.

\section{AUTHOR CONTRIBUTIONS}

EY: Conceptualization, Data curation, Supervision. FD: Conceptualization, Formal Analysis, Writing - original draft, Writing - review \& editing. RA: Conceptualization, Formal Analysis, Writing - original draft, Writing - review \& editing.

4. Ak R, Kurt E, Bahadirli S. Comparison of 2 Risk Prediction Models Specific for COVID-19: The Brescia-COVID Respiratory Severity Scale Versus the Quick COVID-19 Severity Index. Disaster Med Public Health Prep. 2021;15(4):e46-50. https://doi.org/10.1017/ dmp.2021.141

5. Covino M, Matteis G, Santoro M, Sabia L, Simeoni B, Candelli M, et al. Clinical characteristics and prognostic factors in COVID-19 patients aged $\geq 80$ years. Geriatr Gerontol Int. 2020;20(7):704-8. https://doi.org/10.1111/ggi.13960

6. Covino M, De Matteis G, Burzo ML, Russo A, Forte E, Carnicelli A, et al. Predicting in-hospital mortality in COVID-19 older patients with specifically developed scores. J Am Geriatr Soc. 2021;69(1):3743. https://doi.org/10.1111/jgs.16956 
7. Doğanay F, Elkonca F, Seyhan AU, Yılmaz E, Batırel A, Ak R. Shock index as a predictor of mortality among the Covid-19 patients. Am J Emerg Med. 2021;40:106-9. https://doi.org/10.1016/j. ajem.2020.12.053

8. Chalmers S, Khawaja A, Wieruszewski PM, Gajic O, Odeyemi Y. Diagnosis and treatment of acute pulmonary inflammation in critically ill patients: the role of inflammatory biomarkers. World J Crit Care Med. 2019;8(5):59-71. https://doi.org/10.5492/wjccm.v8.i5.59

9. Wang L. C-reactive protein levels in the early stage of COVID-19. Med Mal Infect. 2020;50(4):332-4. https://doi.org/10.1016/j. medmal.2020.03.007

10. Feketea GM, Vlacha V. The diagnostic significance of usual biochemical parameters in coronavirus disease 19 (COVID19): albumin to globulin ratio and CRP to albumin ratio. Front Med (Lausanne). 2020;7:566591. https://doi.org/10.3389/ fmed.2020.566591

11. Zhang J, Wang X, Jia X, Li J, Hu K, Chen G, Wei J, Gong Z, Zhou C, Yu H, Yu M, Lei H, Cheng F, Zhang B, Xu Y, Wang G, Dong W. Risk factors for disease severity, unimprovement, and mortality in COVID-19 patients in Wuhan, China. Clin Microbiol Infect. 2020;26(6):767-72. https://doi.org/10.1016/j.cmi.2020.04.012

12. Wu C, Chen X, Cai Y, Xia J, ZhouX, Xu S, et al. Risk factors associated with acute respiratory distress syndrome and death in patients with Coronavirus Disease 2019 pneumonia in Wuhan, China. JAMA Intern Med. 2020;180(7):934-43. https://doi.org/10.1001/ jamainternmed.2020.0994

13. El-Shabrawy M,AlsadikME, El-Shafei M,Abdelmoaty AA, Alazzouni AS, Esawy MM, etal. Interleukin-6 and C-reactive protein/albumin ratio as predictors of COVID-19 severity and mortality. Egypt J Bronchol. 2021;15(1): 1-7.https://doi.org/10.1186/s43168-021-00054-1

14. Seyhan AU, Doğanay F, Yılmaz E, Aydıner Ö, Ak R, Tekol SD. The comparison of chest CT and RT-PCR during the diagnosis of COVID19. J Clin Med Kaz. 2021;18(1):53-6. https://doi.org/10.23950/ $\mathrm{jcmk} / 9656$

15. DeLong ER, DeLong DM, Clarke-Pearson DL. Comparing the areas under two or more correlated receiver operating characteristic curves: a nonparametric approach. Biometrics. 1988;44(3):83745. PMID: 3203132
16. Fluss R, Faraggi D, Reiser B. Estimation of the Youden index and its associated cutoff point. Biom J. 2005;47(4):458-72. https:// doi.org/10.1002/bimj.200410135

17. Sharma G, Hanania NA, Shim YM. The aging immune system and its relationship to the development of chronic obstructive pulmonary disease. Proc Am Thorac Soc. 2009;6(7):573-80. https://doi. org/10.1513/pats.200904-022RM

18. Dhama K, Patel SK, Kumar R, Rana J, Yatoo MI, Kumar A, et al. Geriatric population during the COVID-19 pandemic: problems, considerations, exigencies, and beyond. Front Public Health. 2020;8:574198. https://doi.org/10.3389/fpubh.2020.574198

19. Yılmaz E, Ak R, Doğanay F. Usefulness of the neutrophil-tolymphocyte ratio in predicting the severity of COVID-19 patients: a retrospective cohort study. Sao Paulo Med J. 2021:S151631802021005019201. https://doi.org/10.1590/15163180.2021.0298.R1.27052021

20. Ragab D, Salah Eldin H, Taeimah M, Khattab R, Salem R. The COVID-19 Cytokine Storm; what we know so far. Front Immunol. 2020;11:1446. https://doi.org/10.3389/fimmu.2020.01446

21. Aziz M, Fatima R, Lee-Smith W, Assaly R. The association of low serumalbumin level with severe COVID-19: a systematic review and meta-analysis. Crit Care. 2020;24(1):255. https://doi.org/10.1186/ s13054-020-02995-3

22. Li B, Yang J, Zhao F, Zhi L, Wang X, Liu L, et al. Prevalence and impact of cardiovascular metabolic diseases on COVID-19 in China. Clin Res Cardiol. 2020;109(5):531-8. https://doi.org/10.1007/ s00392-020-01626-9

23. Mehra MR, Desai SS, Kuy S, Henry TD, Patel AN. Cardiovascular disease, drug therapy, and mortality in COVID-19. N Engl J Med. 2020;382(25):e102. https://doi.org/10.1056/NEJMoa2007621

24. Sarnak MJ, Jaber BL. Pulmonary infectious mortality among patients with end-stage renal disease. Chest. 2001;120(6):1883-7. https:// doi.org/10.1378/chest.120.6.1883

25. Henry BM, Lippi G. Chronic kidney disease is associated with severe coronavirus disease 2019 (COVID-19) infection. Int Urol Nephrol. 2020;52(6):1193-4. https://doi.org/10.1007/s11255020-02451-9 